\title{
Hamiltonian theory for the non-rigid Earth: Semidiurnal terms
}

\author{
J. Getino ${ }^{1}$, J. M. Ferrándiz ${ }^{2}$, and A. Escapa ${ }^{1}$ \\ 1 Grupo de Mecánica Celeste, Facultad de Ciencias, 47005 Valladolid, Spain \\ 2 Departamento de Análisis Matemático y Matemática Aplicada, Universidad de Alicante, 03080 Alicante, Spain
}

Received 15 June 2000 / Accepted 31 January 2001

\begin{abstract}
The purpose of this paper is to determine the contributions to the nutation series arising from the triaxiality of a non-rigid Earth model composed of a rigid mantle and a liquid core. With this aim, the canonical formulation of the rotation of the non-rigid Earth developed by Getino and Ferrándiz is applied in order to study the semidiurnal terms arising from the $C_{22}$ and $S_{22}$ geopotential coefficients. Once the corresponding generating function is calculated, analytical expressions of the Andoyer and figure planes are derived. We also provide numerical nutation series based on the analytical formulae.
\end{abstract}

Key words. celestrial mechanics - earth - method: analytical

\section{Introduction}

The Hamiltonian formalism has been applied by Getino and Ferrándiz to the study of non-rigid Earth models. At present, we have performed a comprehensive treatment of an Earth model composed of an elastic, axis-symmetric mantle, and a liquid core, including the dissipative effects in the core--mantle boundary (CMB) and the delay in the response of the deformation due to the inelasticity of the mantle. The corresponding free problem, with the determination of the free frequencies, is developed in Getino \& Ferrándiz (1997), while the nutation series concerning the forced motion can be found in Getino \& Ferrándiz (1999, 2000a).

We note that these previous studies refer to an axissymmetric Earth model. Thus, the perturbed potential arises from the $J_{2}$ part of the geopotential coefficient which is the main contribution to the nutation series.

However, the Earth's orientation in space nowadays can be determined with great accuracy. As a direct consequence, it is necessary to consider some effects, previously disregarded, which could contribute to the nutation series and which must be taken into account in order to achieve a more complete model.

Particularly, although the Earth is almost to an axissymmetric body, the influence of its triaxiality on the nutation is not negligible. The influence of this effect on nutations was calculated firstly by Kinoshita (1977) for the biggest terms and by Kinoshita \& Souchay (1990) up to

Send offprint requests to: J. Getino,

e-mail: getino@maf .uva.es
0.005 milli arcsec More recently, Folgueira et al. (1998), Souchay et al. (1999) and Bretagnon et al. (1997) have evaluated the coefficients of nutation due to higher parts of the geopotential, obtaining the diurnal and the semidiurnal terms. Notice that all these works assume rigid Earth.

In this paper we begin with the study of the semidiurnals terms coming from the $C_{22}$ and $S_{22}$ geopotential coefficients by considering a non-rigid Earth model and applying the Hamiltonian formalism developed by Getino and Ferrándiz. Due to the small magnitude of these terms, in a first approximation it is enough to consider a rigid mantle, liquid core Earth model, disregarding the effect of the elasticity, whose contribution is negligible.

In Sect. 2 we summarize the formalism applied to a symmetric Earth which will be extended in Sect. 3 to take into account the effect of the triaxiality. Under the Hamiltonian framework the semidiurnal terms are obtained through the generating function, which is studied in Sect. 4. In Sect. 5 the analytical expressions of the nutations of the Andoyer and figure planes are derived. Finally, in Sect. 6 we compute the numerical values of the nutations, making a comparison with the values obtained by Souchay et al. (1999) and Bretagnon et al. (1997) for a rigid Earth model.

\section{The symmetrical two-layered Earth model: An overview}

In this section we give a brief description of the Hamiltonian approach to the free and forced nutations 
of a symmetrical Earth. Further information, as well as a detailed study of this problem, can be found in Getino \& Ferrándiz (1997, 1999, 2000a).

\subsection{Canonical variables and kinetic energy}

We consider an Earth model composed of a mantle and a liquid core. As pointed out in the introduction, we can neglect the effect of the elasticity without harm, and consider the mantle as a rigid layer. Let $O X Y Z$ be a non-rotating inertial frame, $O X_{\mathrm{m}} Y_{\mathrm{m}} Z_{\mathrm{m}}$ the frame of the principal axes of the total Earth rotating with an angular velocity $\boldsymbol{\omega}$ with respect to the inertial frame, and $O X_{\mathrm{c}} Y_{\mathrm{c}} Z_{\mathrm{c}}$ a core fixed frame rotating with angular velocity $\delta \boldsymbol{\omega}$ with respect to the mantle.

With an appropriate definition of the core rotation (Moritz \& Mueller 1987), the angular momentum vectors of mantle and core, $\boldsymbol{L}_{\mathrm{m}}$ and $\boldsymbol{L}_{\mathrm{c}}$, can be expressed in the $O X_{\mathrm{m}} Y_{\mathrm{m}} Z_{\mathrm{m}}$ frame as:

$\boldsymbol{L}_{\mathrm{m}}=\Pi_{\mathrm{m}} \omega, \quad \boldsymbol{L}_{\mathrm{c}}=\Pi_{\mathrm{c}}(\omega+\delta \omega)$,

where $\omega$ and $\delta \omega$ are respectively the corresponding column vectors for $\boldsymbol{\omega}$ and $\delta \boldsymbol{\omega}$ in the $O X_{\mathrm{m}} Y_{\mathrm{m}} Z_{\mathrm{m}}$ frame, and

$\Pi_{\mathrm{m}}=\left(\begin{array}{lll}A_{\mathrm{m}} & 0 & 0 \\ 0 & A_{\mathrm{m}} & 0 \\ 0 & 0 & C_{\mathrm{m}}\end{array}\right), \Pi_{\mathrm{c}}=\left(\begin{array}{lll}A_{\mathrm{c}} & 0 & 0 \\ 0 & A_{\mathrm{c}} & 0 \\ 0 & 0 & C_{\mathrm{c}}\end{array}\right)$.

Let us consider the total angular momentum of the Earth, $\boldsymbol{L}$, which will be:

$\boldsymbol{L}=\boldsymbol{L}_{\mathrm{m}}+\boldsymbol{L}_{\mathrm{c}}=\Pi \omega+\Pi_{\mathrm{c}} \delta \omega$,

where

$\Pi=\Pi_{\mathrm{m}}+\Pi_{\mathrm{c}}=\left(\begin{array}{lll}A & 0 & 0 \\ 0 & A & 0 \\ 0 & 0 & C\end{array}\right)$

is the tensor of inertia of the total Earth. According to this, the components of the total angular momentum and the angular momentum of the core in the $O X_{\mathrm{m}} Y_{\mathrm{m}} Z_{\mathrm{m}}$ frame are:

$\boldsymbol{L}=\left(\begin{array}{c}A \omega_{1}+A_{\mathrm{c}} \delta \omega_{1} \\ A \omega_{2}+A_{\mathrm{c}} \delta \omega_{2} \\ C \omega_{3}+C_{\mathrm{c}} \delta \omega_{3}\end{array}\right), \quad \boldsymbol{L}_{\mathrm{c}}=\left(\begin{array}{c}A_{\mathrm{c}} \omega_{1}+A_{\mathrm{c}} \delta \omega_{1} \\ A_{\mathrm{c}} \omega_{2}+A_{\mathrm{c}} \delta \omega_{2} \\ C_{\mathrm{c}} \omega_{3}+C_{\mathrm{c}} \delta \omega_{3}\end{array}\right)$.

Thus, the kinetic energy is written as:

$$
\begin{aligned}
T_{0} & =\frac{1}{2} \boldsymbol{L}_{\mathrm{m}}^{\mathrm{t}} \Pi_{\mathrm{m}}^{-1} \boldsymbol{L}_{\mathrm{m}}+\frac{1}{2} \boldsymbol{L}_{\mathrm{c}}^{\mathrm{t}} \Pi_{\mathrm{c}}^{-1} \boldsymbol{L}_{\mathrm{c}} \\
& =\frac{1}{2}\left(\boldsymbol{L}-\boldsymbol{L}_{\mathrm{c}}\right)^{\mathrm{t}} \Pi_{\mathrm{m}}^{-1}\left(\boldsymbol{L}-\boldsymbol{L}_{\mathrm{c}}\right)+\frac{1}{2} \boldsymbol{L}_{\mathrm{c}}^{\mathrm{t}} \Pi_{\mathrm{c}}^{-1} \boldsymbol{L}_{\mathrm{c}} .
\end{aligned}
$$

To formulate canonically the kinetic energy, we use the set of canonical variables

$\lambda, \mu, \nu, \Lambda, M, N, \quad \longrightarrow$ for the total Earth,

$\lambda_{\mathrm{c}}, \mu_{\mathrm{c}}, \nu_{\mathrm{c}}, \Lambda_{\mathrm{c}}, M_{\mathrm{c}}, N_{\mathrm{c}}, \longrightarrow$ for the core.
The meaning of the angular variables is shown in Fig. 1. As for the canonical momenta, $N$ is the $O Z_{\mathrm{m}}$ component of the total angular momentum $\boldsymbol{L}, M$ is the magnitude of $\boldsymbol{L}$ and $\Lambda$ is the $O Z$ component of $\boldsymbol{L}$. Similarly for the core, $N_{\mathrm{c}}$ is the $O Z_{\mathrm{m}}$ component of the angular momentum of the core $\boldsymbol{L}_{\mathrm{c}}, M_{\mathrm{c}}$ is the magnitude of $\boldsymbol{L}_{\mathrm{c}}$ and $\Lambda_{\mathrm{c}}$ is the $O Z_{\mathrm{c}}$ component of $\boldsymbol{L}_{\mathrm{c}}$. Thus, by means of the auxiliary angles $\sigma, I, \sigma_{\mathrm{c}}$ and $I_{\mathrm{c}}$, we have the relationships

$$
\begin{array}{ll}
M=|\boldsymbol{L}| & M_{\mathrm{c}}=\left|\boldsymbol{L}_{\mathrm{c}}\right| \\
N=M \cos \sigma & N_{\mathrm{c}}=M_{\mathrm{c}} \cos \sigma_{\mathrm{c}} \\
\Lambda=M \cos I & \Lambda_{\mathrm{c}}=M_{\mathrm{c}} \cos I_{\mathrm{c}}
\end{array}
$$

Furthermore, according to Fig. 1, we can write the components of $\boldsymbol{L}$ and $\boldsymbol{L}_{\mathrm{c}}$ in the $O X_{\mathrm{m}} Y_{\mathrm{m}} Z_{\mathrm{m}}$ frame in terms of the canonical variables as follows

$\boldsymbol{L}=\left(\begin{array}{c}K \sin \nu \\ K \cos \nu \\ N\end{array}\right), \quad \boldsymbol{L}_{\mathrm{c}}=\left(\begin{array}{c}K_{\mathrm{c}} \sin \nu_{\mathrm{c}} \\ -K_{\mathrm{c}} \cos \nu_{\mathrm{c}} \\ N_{\mathrm{c}}\end{array}\right)$

where we have put

$$
\begin{aligned}
K & =\left(M^{2}-N^{2}\right)^{1 / 2}=M \sin \sigma, \\
K_{\mathrm{c}} & =\left(M_{\mathrm{c}}^{2}-N_{\mathrm{c}}^{2}\right)^{1 / 2}=M_{\mathrm{c}} \sin \sigma_{\mathrm{c}} .
\end{aligned}
$$

Finally, introducing (8) in (6) we get the canonical expression of the kinetic energy corresponding to this basic symmetrical Earth model as follows:

$$
\begin{aligned}
T_{0}= & \frac{1}{2\left(A-A_{\mathrm{c}}\right)}\left(K^{2}+\frac{A}{A_{\mathrm{c}}} K_{\mathrm{c}}^{2}\right) \\
& +\frac{1}{2\left(C-C_{\mathrm{c}}\right)}\left(N^{2}-2 N N_{\mathrm{c}}+\frac{C}{C_{\mathrm{c}}} N_{\mathrm{c}}^{2}\right) \\
& +\frac{K K_{\mathrm{c}}}{A-A_{\mathrm{c}}} \cos \left(\nu+\nu_{\mathrm{c}}\right) .
\end{aligned}
$$

\subsection{Dissipative coupling torque}

We introduce the effect of dissipative forces in the boundary mantle-core, including electromagnetic coupling and the effects of the viscosity, following the same assumptions as in Sasao et al. (1980). Thus, as a general expression we can write

$\boldsymbol{t}_{\mathrm{c}}=-\boldsymbol{t}_{\mathrm{m}}=\left(\begin{array}{c}-R \delta \omega_{1}+R^{\prime} \delta \omega_{2} \\ -R \delta \omega_{2}-R^{\prime} \delta \omega_{1} \\ -R^{*} \delta \omega_{3}\end{array}\right)$

where $R, R^{\prime}$ and $R^{*}$ are coupling constants. Note that we have written $R, R^{\prime}, R^{*}$ instead of $K, K^{\prime}, K^{*}$ in the original to avoid confusion in the notation. In particular, the ratio $\eta=R^{\prime} / R$ is nearly zero in viscous coupling and unity in electromagnetic coupling (Sasao et al. 1980). As usual, this torque is characterized by the dimensionless complex coefficient

$\tilde{\Gamma}=\Gamma^{\prime}-i \Gamma$. 


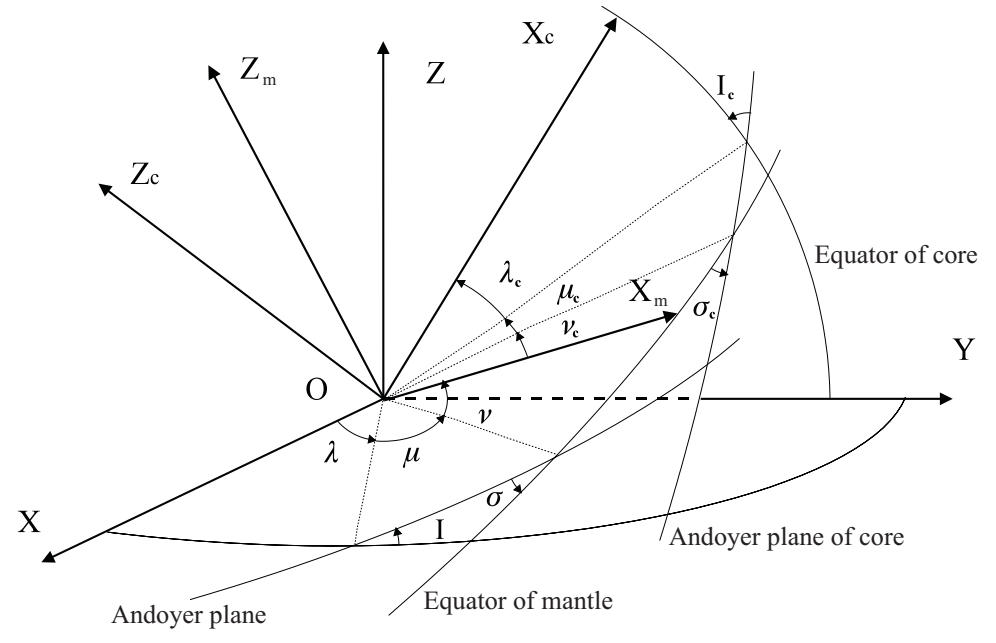

Fig. 1. Canonical variables

These new coefficients are related to the electromagnetic coupling constants $R$ and $R^{\prime}$ by the relationships

$\Gamma^{\prime}=\frac{R^{\prime}}{\Omega A_{\mathrm{m}}}, \quad \Gamma=\frac{R}{\Omega A_{\mathrm{m}}}$.

In the Hamiltonian framework the dissipative effects are formulated by means of the corresponding generalized forces $Q_{p}, Q_{q}$, whose expression, developed in Getino and Ferrándiz (1997), will be given below.

\subsection{Hamiltonian for the symmetrical Earth and first order integration}

According to the previous sections, the Hamiltonian of the system (to the first order) is written as

$H=T_{0}+V_{0}$,

where $V_{0}$ is the perturbing potential coming from the $J_{2}$ geopotential coefficient, and is the same as in Kinoshita (1977) for the rigid Earth, and $T_{0}$ corresponds to the kinetic energy of an axis-symmetric rigid mantle - liquid core model, given by Eq. (10). Note that the generalized forces due to dissipative effects do not appear explicitly into the Hamiltonian (14), but they are taken into account in the corresponding equations of motion (Stiefel \& Scheifele 1971). Now we perform a first order analytical integration of this Hamiltonian by using Hori's perturbation method (1966), following the same procedure as in Kinoshita (1977) for the rigid Earth. This procedure is briefly described here.

First of all, the Hamiltonian (14) is separated into an unperturbed part, $H_{0}$, corresponding to the free motion, and a perturbed part $H_{1}$, for the forced perturbations, in the form

$H=H_{0}+H_{1} \rightarrow\left\{\begin{array}{l}H_{0}=T_{0} \\ H_{1}=V_{0}\end{array}\right.$.

Then, we carry out a canonical transformation of the initial Hamiltonian $H$ into a new one, $H^{*}$, easier to integrate

$H=H_{0}+H_{1} \rightarrow H^{*}=H_{0}^{*}+H_{1}^{*}$, by means of a generating function $W$. In the new Hamiltonian, the unperturbed part is the same as in the old one, $H_{0}^{*}=H_{0}$, and for the new disturbing term we take the secular part of $H_{1}$, that is to say, $H_{1}^{*}=H_{1 \mathrm{sec}}$. We have finally

$$
\begin{aligned}
H_{0}^{*}= & \frac{1}{2 A_{\mathrm{m}}}\left[K^{* 2}+\frac{A}{A_{\mathrm{c}}} K_{\mathrm{c}}^{* 2}\right]+\frac{K^{*} K_{\mathrm{c}}^{*}}{A_{\mathrm{m}}} \cos \left(\nu^{*}+\nu_{\mathrm{c}}^{*}\right) \\
& +\frac{1}{2 C_{\mathrm{m}}}\left[N^{* 2}-2 N^{*} N_{\mathrm{c}}^{*}+\frac{C}{C_{\mathrm{c}}} N_{\mathrm{c}}^{* 2}\right] \\
H_{1}^{*}= & V_{0 \mathrm{sec}}
\end{aligned}
$$

where expression of $V_{0 \text { sec }}$ can be found in Kinoshita (1977). Note that we have used asterisks to indicate the new variables resulting from the canonical transformation. However, in the following, these asterisks will be omitted for the sake of simplicity.

The periodic perturbations (nutations) are obtained through the generating function by means of the equations

$\Delta q=\frac{\partial W}{\partial p^{*}}, \quad \Delta p=-\frac{\partial W}{\partial q^{*}}$

where $q$ stands for the angular variables $\left(\lambda, \mu, \nu, \lambda_{\mathrm{c}}, \mu_{\mathrm{c}}\right.$, $\left.\nu_{\mathrm{c}}\right)$ and $p$ for the conjugated momenta $\left(\Lambda, M, N, \Lambda_{\mathrm{c}}\right.$, $\left.M_{\mathrm{c}}, N_{\mathrm{c}}\right)$. This generating function of the transformation is obtained through

$W=\int\left(H_{1}-H_{1}^{*}\right) \mathrm{d} t=\int H_{1 \mathrm{per}} \mathrm{d} t$

where this integral is performed along the solution of the unperturbed part. This solution, corresponding to the free motion problem, is described in the next subsection.

\subsection{Unperturbed solutions}

The free motion problem is solved by means of the corresponding equations of motion. It is convenient to include the effect of the dissipation in this free motion problem. Thus, the equations of motion must be modified to take 
into account the corresponding generalized forces. These equations are of the form (Stiefel \& Scheifele 1971)

$\dot{q}=\frac{\partial T_{0}}{\partial p}-Q_{p}, \quad \dot{p}=-\frac{\partial T_{0}}{\partial q}+Q_{q}$.

For our study, the required generalized equations of motion are (Getino \& Ferrándiz 1997)

$\dot{\mu}=\partial T_{0} / \partial M-Q_{M}, \quad \dot{\mu}_{\mathrm{c}}=\partial T_{0} / \partial M_{\mathrm{c}}-Q_{M_{\mathrm{c}}}$,

$\dot{\nu}=\partial T_{0} / \partial N-Q_{N}, \quad \dot{\nu}_{\mathrm{c}}=\partial T_{0} / \partial N_{\mathrm{c}}-Q_{N_{\mathrm{c}}}$,

$\dot{N}=-\partial T_{0} / \partial \nu+Q_{\nu}, \quad \dot{N}_{\mathrm{c}}=-\partial T_{0} / \partial \nu_{\mathrm{c}}+Q_{\nu_{\mathrm{c}}}$,

$\dot{M}=-\partial T_{0} / \partial \mu+Q_{\mu}, \dot{M}_{\mathrm{c}}=-\partial T_{0} / \partial \mu_{\mathrm{c}}+Q_{\mu_{\mathrm{c}}}$,

and taking into account the expression of $T_{0}(10)$ we can write

$$
\begin{aligned}
\dot{\mu} & =\frac{M}{A_{\mathrm{m}}}+\frac{M}{A_{\mathrm{m}}} \frac{K_{\mathrm{c}}}{K} \cos \left(\nu+\nu_{\mathrm{c}}\right)-Q_{M}, \\
\dot{\nu} & =-\frac{N}{A_{\mathrm{m}}}+\frac{N-N_{\mathrm{c}}}{C_{\mathrm{m}}}-\frac{N}{A_{\mathrm{m}}} \frac{K_{\mathrm{c}}}{K} \cos \left(\nu+\nu_{\mathrm{c}}\right)-Q_{N}, \\
\dot{N} & =\frac{1}{A_{\mathrm{m}}} K K_{\mathrm{c}} \sin \left(\nu+\nu_{\mathrm{c}}\right)+Q_{\nu}, \\
\dot{M} & =Q_{\mu},
\end{aligned}
$$

for the mantle, and

$$
\begin{aligned}
\dot{\mu}_{\mathrm{c}}= & \frac{A}{A_{\mathrm{c}}} \frac{M_{\mathrm{c}}}{A_{\mathrm{m}}}+\frac{M_{\mathrm{c}}}{A_{\mathrm{m}}} \frac{K}{K_{\mathrm{c}}} \cos \left(\nu+\nu_{\mathrm{c}}\right)-Q_{M_{\mathrm{c}}}, \\
\dot{\nu}_{\mathrm{c}}= & -\frac{A}{A_{\mathrm{c}}} \frac{N_{\mathrm{c}}}{A_{\mathrm{m}}}+\frac{1}{C_{\mathrm{m}}}\left(N-\frac{C}{C_{\mathrm{c}}} N_{\mathrm{c}}\right) \\
& -\frac{N_{\mathrm{c}}}{A_{\mathrm{m}}} \frac{K}{K_{\mathrm{c}}} \cos \left(\nu+\nu_{\mathrm{c}}\right)-Q_{N_{\mathrm{c}}}, \\
\dot{N}_{\mathrm{c}}= & \frac{1}{A_{\mathrm{m}}} K K_{\mathrm{c}} \sin \left(\nu+\nu_{\mathrm{c}}\right)+Q_{\nu_{\mathrm{c}}}, \\
\dot{M}_{\mathrm{c}}= & Q_{\mu_{\mathrm{c}}}
\end{aligned}
$$

for the core. The generalized forces appearing in (23) are obtained in Getino \& Ferrándiz (1997). The expressions of $Q_{i}$ which will be used in the following are

$$
\begin{aligned}
Q_{\mu_{\mathrm{c}}}= & -\frac{R}{A_{\mathrm{m}}} \sin \sigma_{\mathrm{c}}\left[K \cos \left(\nu+\nu_{\mathrm{c}}\right)+\frac{A}{A_{\mathrm{c}}} K_{\mathrm{c}}\right] \\
& +\frac{R^{*}}{C_{\mathrm{m}}} \cos \sigma_{\mathrm{c}}\left[N-\frac{C}{C_{\mathrm{c}}} N_{\mathrm{c}}\right] \\
& -\frac{R^{\prime}}{A_{\mathrm{m}}} \sin \sigma_{\mathrm{c}} K \sin \left(\nu+\nu_{\mathrm{c}}\right), \\
Q_{\nu_{\mathrm{c}}}= & \frac{R^{*}}{C_{\mathrm{m}}}\left[N-\frac{C}{C_{\mathrm{c}}} N_{\mathrm{c}}\right], \\
Q_{N_{\mathrm{c}}}= & -\frac{R}{A_{\mathrm{m}}} \frac{K}{K_{\mathrm{c}}} \sin \left(\nu+\nu_{\mathrm{c}}\right) \\
& +\frac{R^{\prime}}{A_{\mathrm{m}}}\left[\frac{K}{K_{\mathrm{c}}} \cos \left(\nu+\nu_{\mathrm{c}}\right)+\frac{A}{A_{\mathrm{c}}}\right], \\
Q_{\mu}= & Q_{\nu}=Q_{M}=Q_{N}=0 .
\end{aligned}
$$

Now, from (22), (23) and (24) we can proceed to solve the problem. By the definition of conjugated momenta, $N$ and $N_{\mathrm{c}}$ are respectively the third components of $\boldsymbol{L}$ and $\boldsymbol{L}_{\mathrm{c}}$ (see Eqs. (5) and (8)), thus (see Getino 1995b for more details):

$$
\left.\begin{array}{l}
N=C \omega_{3}+C_{\mathrm{c}} \delta \omega_{3} \\
N_{\mathrm{c}}=C_{\mathrm{c}} \omega_{3}+C_{\mathrm{c}} \delta \omega_{3}
\end{array}\right\} \Rightarrow\left\{\begin{array}{l}
\omega_{3}=\frac{N-N_{\mathrm{c}}}{C_{\mathrm{m}}}, \\
\delta \omega_{3}=\frac{C N_{\mathrm{c}}-C_{\mathrm{c}} N}{C_{\mathrm{m}} C_{\mathrm{c}}} .
\end{array}\right.
$$

Now, by means of the equations of motion (22), (23) and (24), and taking into account the expression of $\delta \omega_{3}$ in (25) we have

$\dot{N}-\dot{N}_{\mathrm{c}}=\frac{R^{*}}{C_{\mathrm{m}}}\left(\frac{C}{C_{\mathrm{c}}} N_{\mathrm{c}}-N\right)=R^{*} \delta \omega_{3}$,

and then, from (25), we have

$\dot{\omega}_{3}=\frac{R^{*}}{C_{\mathrm{m}}} \delta \omega_{3}$.

On the other hand, from the second equation of (25), neglecting second order terms (product $K K_{\mathrm{c}}$ in equation of $\dot{N})$ we obtain

$\delta \dot{\omega}_{3}=-\frac{C}{C_{\mathrm{c}}} \frac{R^{*}}{C_{\mathrm{m}}} \delta \omega_{3}$,

and finally

$\delta \omega_{3} \rightarrow 0, \quad \omega_{3} \rightarrow$ constant $=\Omega$.

Thus, taking into account Eqs. (25) and (29) we will write in the following $N=C \Omega$ and $N_{\mathrm{c}}=C_{\mathrm{c}} \Omega$.

With regard to the remainder equations, which provide the free frequencies of the so-called polar motion, we define the variables

$u=M \sin \sigma(\sin \nu+i \cos \nu)=i K \mathrm{e}^{-i \nu}$,

$v=M_{\mathrm{c}} \sin \sigma_{\mathrm{c}}\left(\sin \nu_{\mathrm{c}}-i \cos \nu_{\mathrm{c}}\right)=-i K_{\mathrm{c}} \mathrm{e}^{i \nu_{\mathrm{c}}}$.

With the help of Eqs. (9), (22), (23) and (24) and after some algebra, the time derivatives of variables $u, v$ defined in Eq. (30) can be expressed in the form

$\left(\begin{array}{c}\dot{u} \\ \dot{v}\end{array}\right)=i \mathrm{R}\left(\begin{array}{c}u \\ v\end{array}\right)$,

the matrix $\mathrm{R}$ being

$\mathrm{R}=\left(\begin{array}{cc}r_{1} & r_{2} \\ r_{3}+\Omega \tilde{\Gamma} & r_{4}-\Omega \tilde{\Gamma} \frac{A}{A_{\mathrm{c}}}\end{array}\right)$,

whose coefficients are

$$
\begin{aligned}
& r_{1}=\Omega\left(\frac{A_{\mathrm{c}}}{A_{\mathrm{m}}}+e \frac{A}{A_{\mathrm{m}}}\right), \\
& r_{2}=-\Omega \frac{A}{A_{\mathrm{m}}}(1+e), \\
& r_{3}=\Omega \frac{A_{\mathrm{c}}}{A_{\mathrm{m}}}\left(1+e_{\mathrm{c}}\right), \\
& r_{4}=-\Omega \frac{A}{A_{\mathrm{m}}}\left(1+e_{\mathrm{c}}\right),
\end{aligned}
$$


expressed as functions of the dynamical ellipticities

$e=\frac{C-A}{A}, \quad e_{\mathrm{c}}=\frac{C_{\mathrm{c}}-A_{\mathrm{c}}}{A_{\mathrm{c}}}$,

and $\Gamma$ and $\Gamma^{\prime}$ are the dimensionless constants of dissipation, relative to viscous and electromagnetic coupling. The free frequencies corresponding to the solutions of system (31) are as follows

$\sigma_{1}=m_{1}, \quad \sigma_{2}=m_{2}+i d$,

with

$$
\begin{aligned}
m_{1} & =\Omega \frac{C-A}{A_{\mathrm{m}}}, \\
m_{2} & =-\Omega\left[1+\frac{A}{A_{\mathrm{m}}} \frac{C_{\mathrm{c}}-A_{\mathrm{c}}}{A_{\mathrm{c}}}+\frac{A}{A_{\mathrm{c}}} \Gamma^{\prime}\right], \\
d & =\Omega \frac{A}{A_{\mathrm{c}}} \Gamma,
\end{aligned}
$$

Another useful result, which will be used in the computation of the generating function, is derived from the equations of motion corresponding to the variables $\mu$ and $\nu$ (see Eq. (22)). From these equations we get

$$
\dot{\mu}+\dot{\nu}=\Omega+\frac{M-N}{A_{\mathrm{m}}}\left(1+\frac{K_{\mathrm{c}}}{K} \cos \left(\nu+\nu_{\mathrm{c}}\right)\right) .
$$

According to definition of canonical variables (7), the second term in the r.h.s. of Eq. (37) is of the second order in $\sigma$. Therefore, due to the smallness of variables $\sigma$, $\sigma_{\mathrm{c}} \sim 10^{-6} \mathrm{rad}$ (Nikitina 1990), neglecting this term we obtain the first order the relationship (Getino 1995b; Getino \& Ferrándiz 1997)

$\dot{\mu}+\dot{\nu}=\Omega$.

\section{Effect of the triaxiality of the Earth}

This section is devoted to extending the Hamiltonian (14) corresponding to an axis-symmetric, two-layered Earth, in order to include the effect of the triaxiality. Notice that the Hamiltonian formalism has already been applied to a triaxial, two-layered model by González \& Getino (1997) for the free problem, and by Getino et al. (2000), including the effect of dissipation.

In order to take into account this effect, the Hamiltonian (14) must be transformed in two ways: we include new terms in the perturbing potential corresponding to the geopotential coefficients $C_{2,2}$ and $S_{2,2}$, which characterize the triaxiality of the Earth, and the kinetic energy $T$ is also modified.

\subsection{Triaxial potential $V_{\mathrm{Tr}}$}

According to Kinoshita (1977), the additional potential is

$V_{\operatorname{Tr}}=\frac{G m^{*}}{a^{*^{3}}} \frac{A-B}{4}\left(\frac{a^{*}}{r^{*}}\right)^{3} P_{2}^{2}(\sin \delta) \cos 2 \alpha$.
Following Kinoshita, the angular part in (39) is developed in the form

$$
\begin{aligned}
V_{\operatorname{Tr}}= & \eta k^{\prime} \sum_{i, \tau= \pm 1}\left[\frac{3}{4} \sin ^{2} \sigma B_{i} \cos \left(2 \nu-\tau \Theta_{i}\right)\right. \\
& +\sin \sigma C_{i}(\tau) \cos \left(\mu+2 \nu-\tau \Theta_{i}\right) \\
& \left.+\frac{D_{i}(\tau)}{2} \cos \left(2 \mu+2 \nu-\tau \Theta_{i}\right)\right]
\end{aligned}
$$

In expression (40) we have the coefficients

$k^{\prime}=3 \frac{G m^{*}}{a^{*^{3}}} \frac{2 C-A-B}{2}, \quad \eta=\frac{B-A}{2 C-A-B}$,

$m^{*}$ and $a^{*}$ being respectively the mass and semi-major axis concerning the perturbing body (Moon, Sun), and the functions $B_{i}, C_{i}(\tau)$ and $D_{i}(\tau)$ are given by:

$$
\begin{aligned}
B_{i}= & -\frac{1}{6}\left(3 \cos ^{2} I-1\right) A_{i}^{0)}-\frac{1}{2} \sin 2 I A_{i}^{1)} \\
& -\frac{1}{4} \sin ^{2} I A_{i}^{2)}, \\
C_{i}(\tau)= & -\frac{1}{4} \sin 2 I A_{i}^{0)}+\frac{\tau}{4} \sin I(1+\tau \cos I) A_{i}^{2)} \\
& +\frac{1}{2}(1+\tau \cos I)(-1+2 \tau \cos I) A_{i}^{1)}, \\
D_{i}(\tau)= & -\frac{1}{2} \sin ^{2} I A_{i}^{0)}+\tau \sin I(1+\tau \cos I) A_{i}^{1)} \\
& -\frac{1}{4}(1+\tau \cos I)^{2} A_{i}^{2)}
\end{aligned}
$$

where $\Theta_{i}$ is a linear combination of the orbital variables of Moon and Sun, and numerical values of the coefficients $A_{i}^{j)}$ can be found in Kinoshita (1977), and updated in Kinoshita \& Souchay (1990).

\subsection{Triaxial kinetic energy $T_{T r}$}

The kinetic energy corresponding to a triaxial, twolayered model has the following expression (González \& Getino 1997)

$$
\begin{aligned}
T_{\operatorname{Tr}}= & \frac{M^{2}}{2} \sin ^{2} \sigma\left(\frac{\sin ^{2} \nu}{A_{\mathrm{m}}}+\frac{\cos ^{2} \nu}{B_{\mathrm{m}}}\right)+ \\
& +\frac{M_{\mathrm{c}}^{2}}{2} \sin ^{2} \sigma_{\mathrm{c}}\left(\frac{A \sin ^{2} \nu}{A_{\mathrm{m}} A_{\mathrm{c}}}+\frac{B \cos ^{2} \nu}{B_{\mathrm{m}} B_{\mathrm{c}}}\right)+ \\
& +\frac{1}{2 C_{\mathrm{m}}}\left(N^{2}-2 N N_{\mathrm{c}}+\frac{C}{C_{\mathrm{c}}} N_{\mathrm{c}}^{2}\right)+ \\
& +M M_{\mathrm{c}} \sin \sigma \sin \sigma_{\mathrm{c}}\left(\frac{\cos \nu \cos \nu_{\mathrm{c}}}{B_{\mathrm{m}}}-\frac{\sin \nu \sin \nu_{\mathrm{c}}}{A_{\mathrm{m}}}\right) .
\end{aligned}
$$

After a little re-arrangement this term can be rewritten in a more suitable form

$T_{\operatorname{Tr}}=T_{0}+\Delta_{\operatorname{Tr}} T$

where $T_{0}$ is the kinetic energy for a symmetric Earth given by Eq. (10), and $\Delta_{\operatorname{Tr}} T$ represents the increment due to the 
triaxiality, which has the following expression

$$
\begin{aligned}
\Delta_{\operatorname{Tr}} T= & \frac{A_{\mathrm{m}}-B_{\mathrm{m}}}{2 A_{\mathrm{m}} B_{\mathrm{m}}}\left(M \sin \sigma \cos \nu+M_{\mathrm{c}} \sin \sigma_{\mathrm{c}} \cos \nu_{\mathrm{c}}\right)^{2} \\
& +\frac{A_{\mathrm{c}}-B_{\mathrm{c}}}{2 A_{\mathrm{c}} B_{\mathrm{c}}} M_{\mathrm{c}}^{2} \sin ^{2} \sigma_{\mathrm{c}} \cos ^{2} \nu_{\mathrm{c}}
\end{aligned}
$$

\subsection{Hamiltonian $H_{\operatorname{Tr}}$ and first order integration}

Gathering together the previous results, the Hamiltonian for a triaxial, two-layered Earth is expressed as

$H_{\operatorname{Tr}}=T_{0}+\Delta_{\operatorname{Tr}} T+V_{0}+V_{\operatorname{Tr}}$

It is interesting to break down this Hamiltonian into two parts in the form

$H_{\mathrm{Tr}}=H_{\mathrm{S}}+\Delta_{\operatorname{Tr}} H \rightarrow\left\{\begin{array}{l}H_{\mathrm{S}}=T_{0}+V_{0}, \\ \Delta_{\mathrm{Tr}} H=\Delta_{\mathrm{Tr}} T+V_{\operatorname{Tr}},\end{array}\right.$

where $H_{\mathrm{S}}$ is the Hamiltonian corresponding to a symmetric Earth model, whose study has been described in Sect. 2, and $\Delta_{\operatorname{Tr}} H$ encloses the terms arising from the triaxiality. Due to the small order of magnitude of $\Delta_{\operatorname{Tr}} H$ with respect to $T_{0}$, the triaxiality can be treated as a perturbation. Thus, performing a first order analytical integration described in the previous section, the periodic perturbations are given by the generating function

$W=W_{\mathrm{S}}+W_{\operatorname{Tr}} \rightarrow\left\{\begin{array}{l}W_{\mathrm{S}}=\int\left(V_{0}\right)_{\text {per }} \mathrm{d} t \\ W_{\operatorname{Tr}}=\int\left(\Delta_{\operatorname{Tr}} T+V_{\operatorname{Tr}}\right)_{\text {per }} \mathrm{d} t .\end{array}\right.$

Periodic terms coming from $W_{\mathrm{S}}$, corresponding to the symmetric case, have been studied in Getino \& Ferrándiz (2000a). We will focus our attention on $W_{\operatorname{Tr}}$ to obtain the periodic terms due to the triaxiality.

\section{Generating function $W_{\operatorname{Tr}}$ and first simplifications}

As described in Sect. 2 and 3, the generating function arising from the triaxiality is obtained by integrating the periodic part of $\Delta_{\operatorname{Tr}} H(47)$ over the solutions of $H_{0}$ given in Sect. 1.

In order to get $W_{\operatorname{Tr}}$, a first simplification can be performed due to the smallness of angles $\sigma, \sigma_{\mathrm{c}} \sim 10^{-6} \mathrm{rad}$. Neglecting second order terms in $\sigma$ and $\sigma_{\mathrm{c}}$, in expressions (40) and (45) for $V_{\mathrm{T}_{\mathrm{r}}}$ and $\Delta_{\mathrm{T}_{\mathrm{r}}} T$, the generating function reduces to

$$
\begin{array}{r}
W_{\operatorname{Tr}}=\eta k^{\prime} \sum_{i, \tau= \pm 1} \int\left[\sin \sigma C_{i}(\tau) \cos \left(\mu+2 \nu-\tau \Theta_{i}\right)\right. \\
\left.+\frac{D_{i}(\tau)}{2} \cos \left(2 \mu+2 \nu-\tau \Theta_{i}\right)\right] \mathrm{d} t .
\end{array}
$$

To carry out this integration, we follow the same technique developed in Getino \& Ferrándiz (1997), which is summarized here.

\subsection{Auxiliary integrals}

Let us begin by computing two integrals which will be very useful in the development of this technique. These integrals are written as

$$
\begin{aligned}
& I_{3}=\int M \sin \sigma \cos \left(\mu+2 \nu-\tau \Theta_{i}\right) \mathrm{d} t, \\
& I_{4}=\int M_{\mathrm{c}} \sin \sigma_{\mathrm{c}} \cos \left(\mu+\nu-\nu_{\mathrm{c}}-\tau \Theta_{i}\right) \mathrm{d} t .
\end{aligned}
$$

Let $P$ and $Q$ be new integrals whose imaginary parts are respectively $I_{3}$ and $I_{4}$, that is, $I_{3}=\operatorname{Im}\{P\}, I_{4}=\operatorname{Im}\{Q\}$, then defined by

$P=\int i M \sin \sigma \mathrm{e}^{i \nu} \mathrm{e}^{i h} \mathrm{~d} t$,

$Q=\int i M_{\mathrm{c}} \sin \sigma_{\mathrm{c}} \mathrm{e}^{-i \nu_{\mathrm{c}}} \mathrm{e}^{i h} \mathrm{~d} t$

where we have introduced the notation

$h=\mu+\nu-\tau \Theta_{i}$.

On the other hand, the variables $u, v$ in Eq. (30) can be expressed as

$u=i M \sin \sigma \mathrm{e}^{-i \nu}, \quad v=-i M_{\mathrm{c}} \sin \sigma_{\mathrm{c}} \mathrm{e}^{i \nu_{\mathrm{c}}}$.

From Eqs. (51) and (53) the new integrals are written as

$P=-\int u^{*} \mathrm{e}^{i h} \mathrm{~d} t, \quad Q=\int v^{*} \mathrm{e}^{i h} \mathrm{~d} t$

where $u^{*}, v^{*}$ represent respectively the complex conjugates of $u, v$.

Now let us pay attention to the system of equations for the free motion given by Eq. (31). This system can be transformed into

$$
\left(\begin{array}{l}
\int \dot{u}^{*} \mathrm{e}^{i h} \mathrm{~d} t \\
\int \dot{v}^{*} \mathrm{e}^{i h} \mathrm{~d} t
\end{array}\right)=-i \mathrm{R}^{*}\left(\begin{array}{c}
\int u^{*} \mathrm{e}^{i h} \mathrm{~d} t \\
\int v^{*} \mathrm{e}^{i h} \mathrm{~d} t
\end{array}\right) .
$$

The l.h.s. of Eq. (55) can be integrated by parts, and we obtain

$$
\left(\begin{array}{c}
\int \dot{u}^{*} \mathrm{e}^{i h} \mathrm{~d} t \\
\int \dot{v}^{*} \mathrm{e}^{i h} \mathrm{~d} t
\end{array}\right)=\left(\begin{array}{c}
u^{*} \mathrm{e}^{i h} \\
v^{*} \mathrm{e}^{i h}
\end{array}\right)-i n_{h}\left(\begin{array}{c}
\int u^{*} \mathrm{e}^{i h} \mathrm{~d} t \\
\int v^{*} \mathrm{e}^{i h} \mathrm{~d} t
\end{array}\right),
$$

where, taking into account Eq. (38),

$n_{h}=\frac{\mathrm{d} h}{\mathrm{~d} t}=\dot{\mu}+\dot{\nu}-\tau \dot{\Theta}_{i}=\Omega-\tau n_{i}$.

From Eqs. (51), (55) and (56) we get

$$
\left(\begin{array}{r}
P \\
-Q
\end{array}\right)=\frac{\left(\mathrm{R}^{*}-n_{h} 1\right)^{\alpha}}{\left|\mathrm{R}^{*}-n_{h} 1\right|}\left(\begin{array}{c}
-i u^{*} \mathrm{e}^{i h} \\
-i v^{*} \mathrm{e}^{i h}
\end{array}\right),
$$

where the superscript $\alpha$ stands for the adjoint matrix, and 1 is the unit matrix. As the eigenvalues of matrix $R^{*}$ are the complex conjugates of those of matrix $\mathrm{R}$, being these ones the free frequencies $\sigma_{1}$ and $\sigma_{2}$ given by Eqs. (35) and 
(36), taking into account the expression of matrix $\mathrm{R}$ in Eqs. (32) and (33), we have that

$$
\begin{aligned}
\left|\mathrm{R}^{*}-n_{h} 1\right| & =\left(n_{h}-\sigma_{1}^{*}\right)\left(n_{h}-\sigma_{2}^{*}\right), \\
\left(\mathrm{R}^{*}-n_{h} 1\right)^{\alpha} & =\left(\begin{array}{cc}
c_{11} & c_{12} \\
c_{21} & c_{22}
\end{array}\right) \\
& =\left(\begin{array}{cc}
-n_{h}+r_{4}-\Omega \frac{A}{A_{c}} \tilde{\Gamma}^{*} & -r_{2} \\
-r_{3}-\Omega \tilde{\Gamma}^{*} & -n_{h}+r_{1}
\end{array}\right),
\end{aligned}
$$

then, taking into account Eq. (53), the integrals $P$ and $Q$ are given by

$$
\begin{aligned}
P= & -M \sin \sigma \mathrm{e}^{i(h+\nu)} \frac{c_{11}}{\left(n_{h}-\sigma_{1}^{*}\right)\left(n_{h}-\sigma_{2}^{*}\right)} \\
& +M_{\mathrm{c}} \sin \sigma_{\mathrm{c}} \mathrm{e}^{i\left(h-\nu_{\mathrm{c}}\right)} \frac{c_{12}}{\left(n_{h}-\sigma_{1}^{*}\right)\left(n_{h}-\sigma_{2}^{*}\right)}, \\
Q= & -M \sin \sigma \mathrm{e}^{i(h+\nu)} \frac{c_{21}}{\left(n_{h}-\sigma_{1}^{*}\right)\left(n_{h}-\sigma_{2}^{*}\right)} \\
& +M_{\mathrm{c}} \sin \sigma_{\mathrm{c}} \mathrm{e}^{i\left(h-\nu_{\mathrm{c}}\right)} \frac{c_{22}}{\left(n_{h}-\sigma_{1}^{*}\right)\left(n_{h}-\sigma_{2}^{*}\right)},
\end{aligned}
$$

Finally, taking the imaginary parts in Eq. (60), and neglecting second order terms in ellipticities and dissipation, expressions of integrals $I_{3}, I_{4}$ are obtained in the form

$$
\begin{aligned}
I_{3}= & M \sin \sigma\left[\hat{F}_{1}^{a} \sin (h+\nu)+\hat{F}_{1}^{b} \cos (h+\nu)\right] \\
& +M_{\mathrm{c}} \sin \sigma_{\mathrm{c}}\left[\hat{F}_{2}^{a} \sin \left(h-\nu_{\mathrm{c}}\right)+\hat{F}_{2}^{b} \cos \left(h-\nu_{\mathrm{c}}\right)\right], \\
I_{4}= & M \sin \sigma\left[\hat{G}_{1}^{a} \sin (h+\nu)+\hat{G}_{1}^{b} \cos (h+\nu)\right] \\
& +M_{\mathrm{c}} \sin \sigma_{\mathrm{c}}\left[\hat{G}_{2}^{a} \sin \left(h-\nu_{\mathrm{c}}\right)+\hat{G}_{2}^{b} \cos \left(h-\nu_{\mathrm{c}}\right)\right],
\end{aligned}
$$

where,with the help of the notation

$\hat{f}_{1}=n_{h}-m_{1}=\Omega-\tau n_{i}-m_{1}$,

$\hat{f}_{2}=n_{h}-m_{2}=\Omega-\tau n_{i}-m_{2}$,

the following functions have been defined:

$$
\begin{aligned}
\hat{F}_{1}^{a} & =\frac{n_{h}-r_{4}+\Omega \frac{A}{A_{\mathrm{c}}} \Gamma^{\prime}}{\hat{f}_{1} \hat{f}_{2}}, \\
\hat{F}_{1}^{b} & =-\Omega \Gamma \frac{A}{A_{\mathrm{c}}} \frac{n_{h}-r_{4}-\hat{f}_{2}}{\hat{f}_{1} \hat{f}_{2}^{2}} \\
\hat{G}_{1}^{a} & =\frac{-r_{3}-\Omega \Gamma^{\prime}}{\hat{f}_{1} \hat{f}_{2}} \\
\hat{G}_{1}^{b} & =-\Omega \Gamma \frac{\hat{f}_{2}-\frac{A}{A_{c}} r_{3}}{\hat{f}_{1} \hat{f}_{2}^{2}} \\
\hat{F}_{2}^{a} & =\frac{-r_{2}}{\hat{f}_{1} \hat{f}_{2}}, \\
\hat{F}_{2}^{b} & =\Omega \Gamma \frac{A}{A_{\mathrm{c}}} \frac{r_{2}}{\hat{f}_{1} \hat{f}_{2}^{2}}, \\
\hat{G}_{2}^{a} & =\frac{n_{h}-r_{1}}{\hat{f}_{1} \hat{f}_{2}}, \\
\hat{G}_{2}^{b} & =-\Omega \Gamma \frac{A}{A_{\mathrm{c}}} \frac{n_{h}-r_{1}}{\hat{f}_{1} \hat{f}_{2}^{2}} .
\end{aligned}
$$

\subsection{Generating function}

From expressions (49) and (40), the term of the generating function due to the triaxiality can be divided into two parts

$W_{\operatorname{Tr}}=\int V_{\operatorname{Tr}} \mathrm{d} t=W_{\mathrm{C}}+W_{\mathrm{D}}$,

with

$$
\begin{aligned}
& W_{\mathrm{C}}=\eta k^{\prime} \sum_{i, \tau= \pm 1} C_{i}(\tau) \int \sin \sigma \cos \left(\mu+2 \nu-\tau \Theta_{i}\right) \mathrm{d} t \\
& W_{\mathrm{D}}=\eta k^{\prime} \sum_{i, \tau= \pm 1} \frac{D_{i}(\tau)}{2} \int \cos \left(2 \mu+2 \nu-\tau \Theta_{i}\right) \mathrm{d} t
\end{aligned}
$$

The term $W_{\mathrm{D}}$ is the same as in Kinoshita (1977) for the rigid Earth. Taking into account Eq. (38), it is straightforward to obtain

$W_{\mathrm{D}}=\frac{\eta k^{\prime}}{2} \sum_{i, \tau= \pm 1} \frac{D_{i}(\tau)}{2 \Omega-\tau n_{i}} \sin \left(2 \mu+2 \nu-\tau \Theta_{i}\right)$,

were $n_{i}=\dot{\Theta}_{i}$. Now, by means of integral $I_{3}(61)$, the term $W_{\mathrm{C}}$ is expressed as

$$
\begin{aligned}
W_{\mathrm{C}}= & \eta k^{\prime} \sum_{i, \tau= \pm 1} C_{i}(\tau)\left[\sin \sigma\left(\hat{F}_{1}^{a} \sin a_{1}+\hat{F}_{1}^{b} \cos a_{1}\right)\right. \\
& \left.+\frac{M_{\mathrm{c}}}{M} \sin \sigma_{\mathrm{c}}\left(\hat{F}_{2}^{a} \sin a_{2}+\hat{F}_{2}^{b} \cos a_{2}\right)\right]
\end{aligned}
$$

with the arguments

$a_{1}=\mu+2 \nu-\tau \Theta_{i}, a_{2}=\mu+\nu-\nu_{\mathrm{c}}-\tau \Theta_{i}$.

\section{Forced nutations}

The periodic perturbations, forced nutations, are obtained through the generating function by means of the wellknown relationships

$$
\begin{aligned}
\Delta_{\operatorname{Tr}}(\mu, \nu, \lambda) & =\frac{\partial W_{\operatorname{Tr}}}{\partial(M, N, \Lambda)}, \\
\Delta_{\operatorname{Tr}}(M, N, \Lambda) & =\frac{-\partial W_{\operatorname{Tr}}}{\partial(\mu, \nu, \lambda)} .
\end{aligned}
$$

Next we compute the periodic perturbations of the fundamental planes, that is to say, the plane perpendicular to the angular momentum vector (or Andoyer plane), and the plane perpendicular to the figure axis of the Earth (figure plane or equatorial plane).

\subsection{Nutations of the Andoyer plane}

The longitude of the node and the inclination of this plane are given respectively by $\lambda$ and $I=\cos ^{-1}(\Lambda / M)$. The nutations corresponding to these variables, known as Poisson 
terms, are obtained through the equations (Kinoshita 1977)

$\Delta_{\operatorname{Tr}} \lambda=\frac{-1}{M \sin I} \frac{\partial W_{\operatorname{Tr}}}{\partial I}$

$\Delta_{\operatorname{Tr}} I=\frac{1}{M \sin I}\left(\frac{\partial W_{\operatorname{Tr}}}{\partial \lambda}-\cos I \frac{\partial W_{\operatorname{Tr}}}{\partial \mu}\right)$.

Neglecting second order terms in small parameters, the contribution of these nutations comes from the term $W_{\mathrm{D}}$ of the generating function, which corresponds to the rigid perturbation. Using the angles $\psi=-\lambda, \varepsilon=-I$ and taking into account the relationships between functions $C_{i}(\tau)$ and $D_{i}(\tau)$ (see Kinoshita 1977), the Poisson terms at the first order are as follows

$\Delta_{\operatorname{Tr}}^{\mathrm{D}} \psi=-\eta \frac{k_{0}}{\sin \varepsilon} \sum_{i, \tau= \pm 1} \frac{C_{i}(\tau)}{2 \Omega-\tau n_{i}} \sin \left(2 \phi^{\prime}-\tau \Theta_{i}\right)$

$\Delta_{\operatorname{Tr}}^{\mathrm{D}} \varepsilon=\eta k_{0} \sum_{i, \tau= \pm 1} \frac{C_{i}(\tau)}{2 \Omega-\tau n_{i}} \cos \left(2 \phi^{\prime}-\tau \Theta_{i}\right)$,

with

$k_{0}=\frac{k_{0}^{\prime}}{M} \simeq \frac{3 G m^{*}}{a^{*^{3}} \Omega} \frac{C-A}{A}$,

$\phi^{\prime}=\mu+\nu$.

(1977)

Expressions (71) are the same as those of Kinoshita (1977)
for the rigid Earth. That is, the presence of the liquid core and the effects of dissipation do not influence the nutations of the Andoyer plane, which is consistent with the fact that the motion of the angular momentum axis does not depend on the internal constitution of the Earth (Moritz \& Mueller 1987).

\subsection{Nutations of the figure plane}

The longitude of the node, $\lambda_{\mathrm{f}}$, and the inclination $I_{\mathrm{f}}$ of this plane are given to the first order by (Kinoshita 1977)

$\lambda_{\mathrm{f}}=\lambda+\frac{\sigma}{\sin I} \sin \mu, \quad I_{\mathrm{f}}=I+\sigma \cos \mu$.

Following Kinoshita, the periodic perturbations of the increments $\lambda_{\mathrm{f}}-\lambda, I_{\mathrm{f}}-I$, called Oppolzer terms, are given to the first order by

$$
\begin{aligned}
\Delta_{\operatorname{Tr}}\left(\lambda_{\mathrm{f}}-\lambda\right)= & \frac{1}{\sin I} \frac{1}{M \sin \sigma} \times\left[\operatorname { s i n } \mu \left(\frac{\partial W_{\operatorname{Tr}}}{\partial \nu}\right.\right. \\
& \left.\left.-\frac{\partial W_{\operatorname{Tr}}}{\partial \mu}\right)+\sigma \cos \mu \frac{\partial W_{\operatorname{Tr}}}{\partial \sigma}\right],
\end{aligned}
$$

$$
\begin{aligned}
\Delta_{\operatorname{Tr}}\left(I_{\mathrm{f}}-I\right)= & \frac{1}{M \sin \sigma} \times\left[\operatorname { c o s } \mu \left(\frac{\partial W_{\operatorname{Tr}}}{\partial \nu}\right.\right. \\
& \left.\left.-\frac{\partial W_{\operatorname{Tr}}}{\partial \mu}\right)-\sigma \sin \mu \frac{\partial W_{\operatorname{Tr}}}{\partial \sigma}\right] .
\end{aligned}
$$

Neglecting second order, the contribution to the Oppolzer terms comes from $W_{\mathrm{C}}$. From Eq. (67), after some algebra we finally obtain, in terms of the angles $\psi_{\mathrm{f}}=-\lambda_{\mathrm{f}}, \varepsilon_{\mathrm{f}}=$ $-I_{\mathrm{f}}$, the following expressions

$$
\begin{aligned}
\Delta_{\operatorname{Tr}}\left(\psi_{\mathrm{f}}-\psi\right)= & \frac{\eta k_{0}}{\sin \varepsilon} \sum_{i, \tau= \pm 1} C_{i}(\tau) \\
& \times\left[\hat{F}_{1}^{a} \sin \left(2 \phi^{\prime}-\tau \Theta_{i}\right)\right. \\
& \left.+\hat{F}_{1}^{b} \cos \left(2 \phi^{\prime}-\tau \Theta_{i}\right)\right] \\
\Delta_{\operatorname{Tr}}\left(\varepsilon_{\mathrm{f}}-\varepsilon\right)= & -\eta k_{0} \sum_{i, \tau= \pm 1} C_{i}(\tau) \\
& \times\left[\hat{F}_{1}^{a} \cos \left(2 \phi^{\prime}-\tau \Theta_{i}\right)\right. \\
& \left.-\hat{F}_{1}^{b} \sin \left(2 \phi^{\prime}-\tau \Theta_{i}\right)\right]
\end{aligned}
$$

\subsection{Comparison with the rigid Earth results of Kinoshita (1977)}

As we have pointed out, Poisson terms (71) are the same as in the rigid case. In order to compare Oppolzer terms (75) with those of Kinoshita (1977) for the rigid case, we must translate his results to our notation. Namely, the Oppolzer terms, due to the triaxiality of the Earth, of Kinoshita (1977), can be written as

$$
\begin{aligned}
\left.\Delta_{\operatorname{Tr}}\left(\psi_{\mathrm{f}}-\psi\right)\right)_{\text {rigid }}= & \frac{\eta k_{0}}{\sin \varepsilon} \\
& \times \sum_{i, \tau= \pm 1} \frac{C_{i}(\tau) \sin \left(2 \phi^{\prime}-\tau \Theta_{i}\right)}{\left.\dot{\mu})_{\text {rigid }}+2 \dot{\nu}\right)_{\text {rigid }}-\tau n_{i}}
\end{aligned}
$$

$$
\begin{aligned}
\left.\Delta_{\operatorname{Tr}}\left(\varepsilon_{f}-\varepsilon\right)\right)_{\text {rigid }}= & -\eta k_{0} \\
& \times \sum_{i, \tau= \pm 1} \frac{C_{i}(\tau) \cos \left(2 \phi^{\prime}-\tau \Theta_{i}\right)}{\left.\dot{\mu})_{\text {rigid }}+2 \dot{\nu}\right)_{\text {rigid }}-\tau n_{i}}
\end{aligned}
$$

where $\dot{\mu})_{\text {rigid }}$ and $\left.\dot{\nu}\right)_{\text {rigid }}$ are given by (see Kinoshita 1977)

$\left.\dot{\mu})_{\text {rigid }} \simeq \frac{C}{A} \Omega, \dot{\nu}\right)_{\text {rigid }} \simeq-\frac{C-A}{A} \Omega$.

From Eqs. (75) and (76) it follows that Oppolzer terms are substantially different for the rigid and non-rigid cases. On the one hand, in the non-rigid case there are in-phase and out-of-phase terms. Out-of-phase terms come from the function $\hat{F}_{1}^{b}$, that, according to Eq. (63), is produced by 
the dissipation at the CMB (through the coefficients $\Gamma$ ). On the other hand, amplitudes of in-phase terms, given by $\hat{F}_{1}^{a}$, are not the same as in the rigid case.

We can check that our non-rigid model coincides with that of Kinoshita (1977) when removing the non-rigidity of the Earth. With this aim, we must take into account that to convert our model into the rigid one the next steps must be followed. Firstly, in the rigid Earth there is no dissipation of electromagnetic or viscous origin, so we must take $\tilde{\Gamma}=0$. Secondly, the rigid Earth is composed of only one layer, the mantle, therefore we must take $e_{\mathrm{c}}=0$ and $A_{\mathrm{m}}=A$. Bearing these considerations in mind we arrive at

$$
\begin{aligned}
& \left.\hat{F}_{1}^{a}\right)_{\text {rigid }}=\left(\Omega-\tau n_{i}-\Omega \frac{C-A}{A}\right)^{-1}, \\
& \left.\hat{F}_{b}^{a}\right)_{\text {rigid }}=0,
\end{aligned}
$$

that, with the help of Eq. (77), can be re-written as

$$
\begin{aligned}
& \left.\left.\left.\hat{F}_{1}^{a}\right)_{\text {rigid }}=(\dot{\mu})_{\text {rigid }}+2 \dot{\nu}\right)_{\text {rigid }}-\tau n_{i}\right)^{-1}, \\
& \left.\hat{F}_{b}^{a}\right)_{\text {rigid }}=0 .
\end{aligned}
$$

Substituting these functions into Eqs. (75) we recover the Oppolzer terms given by Kinoshita (1977), Eqs. (76).

In order to gain more insight into the difference between the rigid and non-rigid situations, let us introduce the ratio between the in-phase amplitude for the rigid and non-rigid nutations corresponding to the same argument $\left(2 \phi^{\prime}-\tau \Theta_{i}\right)$. Taking into account Eqs. (36), (62), (63) and (79) we obtain

$$
\begin{array}{r|}
\left.\frac{\hat{F}_{1}^{a}}{\left.\hat{F}_{1}^{a}\right)_{\text {rigid }}}\right|_{2 \phi^{\prime}-\tau \Theta_{i}}=\frac{\left(1-e-\tau n_{i} / \Omega\right)}{\left(1-A / A_{\mathrm{m}} e-\tau n_{i} / \Omega\right)} \\
\times \frac{\left[1+A / A_{\mathrm{m}}\left(1+e_{\mathrm{c}}\right)-\tau n_{i} / \Omega\right]}{\left(2+A / A_{\mathrm{m}} e_{\mathrm{c}}-\tau n_{i} / \Omega\right)} .
\end{array}
$$

This equation shows that the ratio between the in-phase amplitudes for the non-rigid and rigid models is a function of some Earth parameters and of the frequency $n_{i}$ corresponding to each term. It is important to note that due to the functional dependence in Eq. (80) and the values of $n_{i}$, there is no possibility of having small divisors, as happens to the analogous ratio for the usual long-period terms coming from $J_{2}$. That is, there is no noticeable resonant effect. The former ratio varies very slightly in the band of the disturbing frequencies $n_{i}$ and is nearly constant. A straightforward Taylor expansion of this ratio with respect to the ellipticities and the frequencies $n_{i}$ provides that its value deviates from 1 in about $A_{\mathrm{c}} / 2 A_{\mathrm{m}}$. This last coefficient is a common factor of terms in $n_{i}$ and the ellipticities, that have therefore a small effect.

The ratio given by Eq. (80) is not a "transfer function" (the ratio of the whole amplitudes of non-rigid and rigid nutations for each period, gathering both Poisson and Oppolzer terms). The true expression of the transfer function can easily be computed from Eqs. (83), (76) and (71). This transfer function is frequency-dependent from the mathematical perspective, although in practice is nearly equal to $1+A_{\mathrm{c}} / A_{\mathrm{m}} \simeq 1.123$ in the band of the relevant disturbing frequencies.

\subsection{Reference to Greenwich prime meridian}

Note that the angle $\phi^{\prime}=\mu+\nu$ refers to the principal axis of the Earth corresponding to the minimum moment of inertia, according to the definition of the Andoyer angle $\nu$ (see Kinoshita 1977; Getino 1995a). The angle $\phi$ of sidereal rotation of the Earth, referred to the Greenwich prime meridian, is obtained by a phase shift in angle $\phi^{\prime}$ (Bretagnon et al. 1997). Thus, we can write a relationship of the form

$2 \phi=2 \phi^{\prime}+g$.

This phase shift is related to the geopotential coefficients $J_{2}, C_{2,2}$ and $S_{2,2}$ by expressions

$\eta \cos g=\frac{2 C_{2,2}}{J_{2}}, \quad \eta \sin g=\frac{2 S_{2,2}}{J_{2}}$.

Finally, introducing Eq. (82) in expressions (71) and (75) we get the following expressions for the nutations of the figure axis

$$
\begin{aligned}
\Delta_{\operatorname{Tr}} \varepsilon_{\mathrm{f}}= & \frac{2 k_{0}}{J_{2}} \sum_{i, \tau= \pm 1} C_{i}(\tau)\left[\frac{1}{2 \Omega-\tau n_{i}}-\hat{F}_{1}^{a}\right] \\
& \times\left[C_{2,2} \cos \left(2 \phi-\tau \Theta_{i}\right)-S_{2,2} \sin \left(2 \phi-\tau \Theta_{i}\right)\right] \\
& +\frac{2 k_{0}}{J_{2}} \sum_{i, \tau= \pm 1} C_{i}(\tau) \hat{F}_{1}^{b} \\
& \times\left[C_{2,2} \sin \left(2 \phi-\tau \Theta_{i}\right)+S_{2,2} \cos \left(2 \phi-\tau \Theta_{i}\right)\right]
\end{aligned}
$$

$$
\begin{aligned}
\Delta_{\operatorname{Tr}} \psi_{\mathrm{f}}= & \frac{-2 k_{0}}{J_{2} \sin \varepsilon} \sum_{i, \tau= \pm 1} C_{i}(\tau)\left[\frac{1}{2 \Omega-\tau n_{i}}-\hat{F}_{1}^{a}\right] \\
& \times\left[C_{2,2} \sin \left(2 \phi-\tau \Theta_{i}\right)+S_{2,2} \cos \left(2 \phi-\tau \Theta_{i}\right)\right] \\
& -\frac{2 k_{0}}{J_{2} \sin \varepsilon} \sum_{i, \tau= \pm 1} C_{i}(\tau) \hat{F}_{1}^{b} \\
& \times\left[C_{2,2} \cos \left(2 \phi-\tau \Theta_{i}\right)-S_{2,2} \sin \left(2 \phi-\tau \Theta_{i}\right)\right]
\end{aligned}
$$




\section{Nutation series}

We proceed in this section to a numerical computation of the forced nutations given by Eqs. (71) for the angular momentum axis and (83) for the figure axis. First of all we take the following numerical values from the IERS standards (McCarthy 1996)

\begin{tabular}{|c|c|}
\hline Parameter & IERS standards \\
\hline$\Omega$ & $7.29211510^{-5} \mathrm{rads}^{-1}$ \\
\hline$\varepsilon$ & $84381^{\prime \prime} 412$ \\
\hline$J_{2}$ & $1082.635910^{-6}$ \\
\hline$C_{2,2}$ & $1.57441010^{-6}$ \\
\hline$S_{2,2}$ & $-0.90375710^{-6}$ \\
\hline
\end{tabular}

The nutations depend on a set of parameters characterizing the Earth model used, through coefficient $r_{4}, \Gamma$ and functions $\hat{f}_{1}, \hat{f}_{2}$ appearing in definition of functions $F_{1}^{a, b}$, as well as the coefficients $k_{0 \mathrm{M}}$ and $k_{0 \mathrm{~S}}$ of the perturbing potential. Analytical formulae of the forced nutations (71) and (83) can be expressed as functions of the following set of basic Earth parameters (BEP)

$\left.\left.B E P=\left\{P_{\mathrm{CW}}, P_{\mathrm{FCN}}, A_{\mathrm{c}} / A_{\mathrm{m}}, \Gamma, k_{0}\right)_{\mathrm{M}}, k_{0}\right)_{\mathrm{S}}\right\}$,

where $P_{\mathrm{CW}}$ and $P_{\mathrm{FCN}}$ are respectively the periods corresponding to the free frequencies $m_{1}$ and $m_{2}(36)$, that is to say,

$P_{\mathrm{CW}}=\left[e \frac{A}{A_{\mathrm{m}}}\right]^{-1}$,

$P_{\mathrm{FCN}}=\left[\frac{A}{A_{\mathrm{m}}} e_{\mathrm{c}}+\frac{A}{A_{\mathrm{c}}} \Gamma^{\prime}\right]^{-1}$,

$A_{\mathrm{c}} / A_{\mathrm{m}}$ is the ratio between principal moments of core and mantle, $\Gamma$ is the coefficient of the dissipation and $\left.\left.k_{0}\right)_{\mathrm{M}}, k_{0}\right)_{\mathrm{S}}$ are the coefficients of the perturbing potential (72) for the Moon and Sun. Finally, by means of Eqs. (33), (36), (57), (62), (63) and (84) we get the expressions

$$
\begin{aligned}
& \hat{F}_{1}^{a}=\frac{2+A_{\mathrm{c}} / A_{\mathrm{m}}+P_{\mathrm{FCN}}^{-1}-\tau n_{i} / \Omega}{\Omega\left(1-P_{\mathrm{CW}}^{-1}-\tau n_{i} / \Omega\right)\left(2+P_{\mathrm{FCN}}^{-1}-\tau n_{i} / \Omega\right)}, \\
& \hat{F}_{1}^{b}=\frac{-\Gamma\left(1+A_{\mathrm{c}} / A_{\mathrm{m}}\right)}{\Omega\left(1-P_{\mathrm{CW}}^{-1}-\tau n_{i} / \Omega\right)\left(2+P_{\mathrm{FCN}}^{-1}-\tau n_{i} / \Omega\right)} .
\end{aligned}
$$

Values of BEP, taken from Getino and Ferrándiz (2000b), are the following

\begin{tabular}{lr}
\hline Parameter & Getino and Ferrándiz \\
\hline$P_{\mathrm{CW}}$ & 400.7 days \\
$P_{\mathrm{FCN}}$ & 432.94 days \\
$A_{\mathrm{c}} / A_{\mathrm{m}}$ & 0.123234 \\
$\Gamma$ & $4.110^{-6}$ \\
$\left.k_{0}\right)_{\mathrm{M}}$ & $7567^{\prime \prime} .870647 / \mathrm{Jcy}$ \\
$\left.k_{0}\right)_{\mathrm{S}}$ & $3474^{\prime \prime} .613747 / \mathrm{Jcy}$ \\
\hline
\end{tabular}

With these parameters the amplitudes of the nutation series can be evaluated. First of all, we show in Table 1 the amplitudes corresponding to the dissipative terms in obliquity (depending on function $\hat{F}_{1}^{b}$ ), where only the main terms are listed. As expected, these values are very small, so that the effect of the dissipation on the semi-diurnal terms can be disregarded in the future.

Main amplitudes of non-dissipative terms are listed in Tables 2 (obliquity) and 3 (longitude), where we have included the amplitudes for angular momentum axis (Poisson terms) and figure axis. Finally in Tables 4 and 5 we compare our results for the amplitudes of the figure axis with those of Bretagnon et al. (1997) and Souchay et al. (1999) for the rigid Earth.

Table 1. Semi-diurnal terms: nutations in obliquity. Dissipative terms (Unit $=\mu$ as $)$

\begin{tabular}{rrrrr|r|r|r|r|}
\hline \multicolumn{7}{c|}{ Argument } & \multicolumn{2}{c|}{ Figure Axis } \\
\hline$\phi$ & $l_{\mathrm{M}}$ & $l_{\mathrm{S}}$ & $F$ & $D$ & $\Omega$ & $C_{22}(\sin )$ & $S_{22}(\cos )$ \\
\hline 2 & 0 & 0 & 0 & 0 & 0 & .029 & -.016 \\
2 & 0 & 0 & 0 & 0 & -1 & .003 & -.002 \\
2 & 0 & 0 & -2 & 2 & -2 & -.009 & .005 \\
2 & -1 & 0 & 0 & 0 & 0 & .001 & -.001 \\
2 & 0 & 0 & -2 & 0 & -2 & -.024 & .013 \\
2 & 0 & 0 & -2 & 0 & -1 & -.004 & .002 \\
2 & -1 & 0 & -2 & 0 & -2 & -.004 & .002 \\
\hline
\end{tabular}

Acknowledgements. This work has been partially supported by Spanish Projects CICYT, Project No. ESP97-1816-C0402 and Junta de Castilla y León, Project No. VA11/99, and Spanish Projects I+D+I, Project No. AYA2000 1787. A. Escapa has been fully supported by a F.P.I. grant of the Junta de Castilla y León.

The authors thank the anonymous referee for his valuable suggestions. 
Table 2. Semi-diurnal terms: nutations in obliquity (Unit $=\mu$ as)

\begin{tabular}{|c|c|c|c|c|c|c|c|c|c|c|c|}
\hline \multicolumn{6}{|c|}{ Argument } & Period & \multicolumn{2}{|c|}{ Angular Momentum } & \multicolumn{2}{|c|}{ Figure Axis } & Alias \\
\hline$\phi$ & $l_{\mathrm{M}}$ & $l_{\mathrm{S}}$ & $F$ & $D$ & $\Omega$ & (days) & $C_{22}(\cos )$ & $S_{22}(\sin )$ & $C_{22}(\cos )$ & $S_{22}(\sin )$ & period \\
\hline 2 & 0 & 0 & 0 & 0 & 0 & .498634 & 12.670 & 7.273 & -14.297 & -8.207 & $\infty$ \\
\hline 2 & 0 & 0 & 0 & 0 & -1 & .498598 & 1.717 & .986 & -1.938 & -1.112 & -6798.36 \\
\hline 2 & 0 & 0 & -2 & 2 & -2 & .500000 & -4.202 & -2.412 & 4.768 & 2.737 & 182.62 \\
\hline 2 & 0 & -1 & -2 & 2 & -2 & .500685 & -.246 & -.141 & .280 & .161 & 121.75 \\
\hline 2 & -1 & 0 & 0 & 0 & 0 & .507826 & .721 & .414 & -.844 & -.485 & 27.55 \\
\hline 2 & 1 & 0 & -2 & 0 & -2 & .507985 & .259 & .148 & -.304 & -.174 & 27.09 \\
\hline 2 & 0 & 0 & -2 & 0 & -2 & .517526 & -9.351 & -5.367 & 11.385 & 6.535 & 13.66 \\
\hline 2 & 0 & 0 & -2 & 0 & -1 & .517569 & -1.763 & -1.012 & 2.147 & 1.232 & 13.63 \\
\hline 2 & 1 & 0 & -2 & -2 & -2 & .526074 & -.345 & -.198 & .435 & .250 & 9.56 \\
\hline 2 & -1 & 0 & -2 & 0 & -2 & .527441 & -1.824 & -1.047 & 2.312 & 1.327 & 9.13 \\
\hline 2 & -1 & 0 & -2 & 0 & -1 & .527474 & -.344 & -.197 & .436 & .250 & 9.12 \\
\hline 2 & 0 & 0 & -2 & -2 & -2 & .536299 & -.296 & -.170 & .389 & .223 & 7.10 \\
\hline 2 & -2 & 0 & -2 & 0 & -2 & .537720 & -.246 & -.141 & .325 & .186 & 6.86 \\
\hline 2 & 0 & 0 & 0 & 0 & 1 & .498671 & -.251 & -.144 & .283 & .162 & 6798.36 \\
\hline 2 & 0 & 0 & 2 & -2 & 2 & .497277 & .179 & .103 & -.201 & -.115 & -182.62 \\
\hline 2 & 1 & 0 & 0 & 0 & 0 & .489770 & .695 & .399 & -.757 & -.435 & -27.55 \\
\hline 2 & 0 & 0 & 2 & 0 & 2 & .481074 & .374 & .214 & -.393 & -.225 & -13.66 \\
\hline 2 & 0 & 0 & 2 & 0 & 1 & .481036 & .239 & .137 & -.251 & -.144 & -13.63 \\
\hline
\end{tabular}

Table 3. Semi-diurnal terms: nutations in longitude (Unit $=\mu$ as)

\begin{tabular}{|c|c|c|c|c|c|c|c|c|c|c|c|}
\hline \multicolumn{6}{|c|}{ Argument } & Period & \multicolumn{2}{|c|}{ Angular Momentum } & \multicolumn{2}{|c|}{ Figure Axis } & \multirow{2}{*}{$\begin{array}{c}\text { Alias } \\
\text { period } \\
\end{array}$} \\
\hline$\phi$ & $l_{\mathrm{M}}$ & $l_{\mathrm{S}}$ & $F$ & $D$ & $\Omega$ & (days) & $C_{22}(\sin )$ & $S_{22}(\cos )$ & $C_{22}(\sin )$ & $S_{22}(\cos )$ & \\
\hline 2 & 0 & 0 & 0 & 0 & 0 & .498634 & -31.853 & 18.285 & 35.944 & -20.632 & $\infty$ \\
\hline 2 & 0 & 0 & 0 & 0 & -1 & .498598 & -4.318 & 2.478 & 4.872 & -2.796 & -6798.36 \\
\hline 2 & 0 & 0 & -2 & 2 & -2 & .500000 & 10.565 & -6.064 & -11.987 & 6.881 & 182.62 \\
\hline 2 & 0 & -1 & -2 & 2 & -2 & .500685 & .620 & -.355 & -.705 & .404 & 121.75 \\
\hline 2 & -1 & 0 & 0 & 0 & 0 & .507826 & -1.814 & 1.041 & 2.124 & -1.219 & 27.55 \\
\hline 2 & 1 & 0 & -2 & 0 & -2 & .507985 & -.652 & .374 & .764 & -.438 & 27.09 \\
\hline 2 & 0 & 0 & -2 & 0 & -2 & .517526 & 23.509 & -13.494 & -28.622 & 16.430 & 13.66 \\
\hline 2 & 0 & 0 & -2 & 0 & -1 & .517569 & 4.433 & -2.545 & -5.399 & 3.099 & 13.63 \\
\hline 2 & 1 & 0 & -2 & -2 & -2 & .526074 & .869 & -.498 & -1.095 & .628 & 9.56 \\
\hline 2 & -1 & 0 & -2 & 0 & -2 & .527441 & 4.587 & -2.633 & -5.812 & 3.336 & 9.13 \\
\hline 2 & -1 & 0 & -2 & 0 & -1 & .527474 & .864 & -.496 & -1.096 & .629 & 9.12 \\
\hline 2 & 0 & 0 & -2 & -2 & -2 & .536299 & .744 & -.427 & -.978 & .561 & 7.10 \\
\hline 2 & -2 & 0 & -2 & 0 & -2 & .537720 & .618 & -.355 & -.817 & .469 & 6.86 \\
\hline 2 & 0 & 0 & 0 & 0 & 1 & .498671 & .631 & -.362 & -.712 & .408 & 6798.36 \\
\hline 2 & 0 & 0 & 2 & -2 & 2 & .497277 & -.452 & .259 & .507 & -.291 & -182.62 \\
\hline 2 & 1 & 0 & 0 & 0 & 0 & .489770 & -1.749 & 1.004 & 1.905 & -1.093 & -27.55 \\
\hline 2 & 0 & 0 & 2 & 0 & 2 & .481074 & -.940 & .539 & .988 & -.567 & -13.66 \\
\hline 2 & 0 & 0 & 2 & 0 & 1 & .481036 & -.602 & .345 & .633 & -.363 & -13.63 \\
\hline
\end{tabular}

Table 4. Comparison with rigid Earth theories. Figure axis. Obliquity (Unit $=\mu$ as)

\begin{tabular}{rrrr|r|r|r|r|rrrrrr|}
\hline \multicolumn{10}{c|}{ Argument } & Period & \multicolumn{2}{c}{ Bretagnon et al. } & \multicolumn{2}{c}{ Souchay et al. } & Authors \\
\hline$\phi$ & $l_{\mathrm{M}}$ & $l_{\mathrm{S}}$ & $F$ & $D$ & $\Omega$ & $($ days $)$ & $\Delta \varepsilon_{\mathrm{f}}(\cos )$ & $\Delta \varepsilon_{\mathrm{f}}(\sin )$ & $\Delta \varepsilon_{\mathrm{f}}(\cos )$ & $\Delta \varepsilon_{\mathrm{f}}(\sin )$ & $\Delta \varepsilon_{\mathrm{f}}(\cos )$ & $\Delta \varepsilon_{\mathrm{f}}(\sin )$ \\
\hline 2 & -1 & 0 & -2 & 0 & -2 & .527441 & 2.05 & 1.17 & 2.06 & 1.18 & 2.312 & 1.327 \\
2 & 0 & 0 & -2 & 0 & -1 & .517569 & 1.90 & 1.09 & 1.88 & 1.08 & 2.147 & 1.232 \\
2 & 0 & 0 & -2 & 0 & -2 & .517526 & 10.08 & 5.79 & 9.89 & 5.68 & 11.385 & 6.535 \\
2 & -1 & 0 & 0 & 0 & 0 & .507826 & -0.74 & -0.43 & -0.72 & -0.42 & -.844 & -.485 \\
2 & 0 & 0 & -2 & 2 & -2 & .500000 & 4.22 & 2.42 & 4.12 & 2.36 & 4.768 & 2.737 \\
2 & 0 & 0 & 0 & 0 & 0 & .498634 & -12.67 & -7.27 & -12.40 & -7.12 & -14.297 & -8.207 \\
2 & 0 & 0 & 0 & 0 & -1 & .498598 & -1.72 & -0.99 & -1.66 & -0.95 & -1.938 & -1.112 \\
\hline
\end{tabular}


Table 5. Comparison with rigid Earth theories. Figure axis. Longitude (Unit $=\mu$ as)

\begin{tabular}{|c|c|c|c|c|c|c|c|c|c|c|c|c|}
\hline \multicolumn{6}{|c|}{ Argument } & Period & \multicolumn{2}{|c|}{ Bretagnon et al. } & \multicolumn{2}{|c|}{ Souchay et al } & \multicolumn{2}{|c|}{ Authors } \\
\hline$\phi$ & $l_{\mathrm{M}}$ & $l_{\mathrm{S}}$ & $F$ & $D$ & $\Omega$ & (days) & $\Delta \psi_{\mathrm{f}}(\sin )$ & $\Delta \psi_{\mathrm{f}}(\cos )$ & $\Delta \psi_{\mathrm{f}}(\sin )$ & $\Delta \psi_{\mathrm{f}}(\cos )$ & $\Delta \psi_{\mathrm{f}}(\sin )$ & $\Delta \psi_{\mathrm{f}}(\cos )$ \\
\hline 2 & -1 & 0 & -2 & $\overline{0}$ & $\overline{-2}$ & .527441 & -5.15 & 2.96 & -5.10 & 2.93 & -5.812 & 3.336 \\
\hline 2 & 0 & 0 & -2 & 0 & 1 & .517569 & -4.32 & 2.48 & -4.71 & 2.70 & -5.399 & 3.099 \\
\hline 2 & 0 & 0 & -2 & 0 & -2 & .517526 & -25.36 & 14.55 & -25.53 & 14.65 & -28.622 & 16.430 \\
\hline 2 & -1 & 0 & 0 & 0 & 0 & .507826 & 1.88 & -1.08 & 1.81 & -1.04 & 2.124 & -1.219 \\
\hline 2 & 0 & 0 & -2 & 2 & -2 & .500000 & -10.63 & 6.10 & -10.45 & 6.00 & -11.987 & 6.881 \\
\hline 2 & 0 & 0 & 0 & 0 & 0 & .498634 & 31.85 & -18.28 & 31.82 & -18.24 & 35.944 & -20.632 \\
\hline 2 & 0 & 0 & 0 & 0 & -1 & .498598 & 4.32 & -2.48 & 4.28 & -2.45 & 4.872 & -2.796 \\
\hline
\end{tabular}

\section{References}

Bretagnon, P., Rocher, P., \& Simon, J. L. 1997, A\&A, 319, 305 Folgueira, M., Souchay, J., \& Kinoshita, H. 1998, Celest. Mech., 69,373

Getino, J. 1995a, Geophys. J. Int., 120, 693

Getino, J. 1995b, Geophys. J. Int., 122, 803

Getino, J., \& Ferrándiz, J. M. 1995, Celest. Mech., 61, 117

Getino, J., \& Ferrándiz, J. M. 1997, Geophys. J. Int., 130, 326

Getino, J., \& Ferrándiz, J. M. 1999, MNRAS Lett., 306(4), L45

Getino, J., \& Ferrándiz, J. M. 2000a, Geophys. J. Int., 142(3), 703

Getino, J., \& Ferrándiz, J. M. 2000b, Forced nutations of a two layers Erath model, MNRAS, submitted

Getino, J., González, A. B., \& Escapa, A. 2000, Celest. Mech., $76(1), 1$
González, A. B., \& Getino, J. 1997, Celest. Mech., 68, 139

Hori, G. 1966, Publ. Astron. Soc. Jpn., 24, 423

Kinoshita, H. 1977, Celest. Mech., 15, 277

Kinoshita, H., \& Souchay, J. 1990, Celest. Mech., 48, 187

McCarthy, D. 1996, IERS Conventions

Moritz, H., \& Mueller, I. 1987, Earth Rotation, Ungar, New York

Nikitina, L. V. 1990, Geomagn. Aeron., 30, 702

Sasao, T., Okubo, S., \& Saito, M. 1980, Proc. IAU Symp., 78, 165

Stiefel, E. L. \& Scheifele, G. 1971, Linear and regular Celestial Mechanics (Springer, New York)

Souchay, J., Losley, B., Kinoshita, H., \& Folgueira, M. 1999, A\&AS, 135, 111 